\title{
Energy Efficiency of Massive-MIMO Heterogeneous Network
}

\author{
Hui Bao ${ }^{\mathrm{a}}$, PengboFang ${ }^{\mathrm{b}}$
}

\author{
School of electrical and Electronic Engineering,North China Electric Power University,Baoding \\ 071000, China. \\ a1641601307@qq.com, ${ }^{\mathrm{b}} 1007962758 @ q q . c o m$
}

Keywords: Massive MIMO, heterogeneous network, energy efficiency, JointSpatial Division andMultiplexing

\begin{abstract}
Massivemassive multiple-input multiple-output (MIMO) and heterogeneous networks are two Potential technologies in the 5G. The application of Massive-MIMO to heterogeneous network can effectively eliminate the interference and improve energy efficiency.In this paper, the network is optimized by using JointSpatial Division and Multiplexing (JSDM) and zero forcing algorithm, analyzing the spectrum efficiency and energy efficiency of the heterogeneous network.The relationship between the energy efficiency and the number of antennas and the transmission power of the base station are considered by simulation.
\end{abstract}

\section{Introduction}

With the rapid development of information and communication technology, the corresponding energy consumption is increasing rapidly. Reducing system energy consumption is getting more and more attention. In the next generation mobile communication system, the deployment of heterogeneous networks and large-scaleantenna technology will be widely used[1][2]. The so-called heterogeneous network is the macro cellular base station and low power nodes in a region of common network, this network can get higher spectrum utilization rate and higher Qos.However, at the same time, the introduction of low power nodes, making the system of interference sources and energy consumption also increase.Therefore,how to reduce the system energy consumption and improve energy efficiency is a hot issue in the future.Massive MIMO technology can greatly reduce the transmission power of the base station and guarantee Qos,soas to improve the performance index of system.

This paper considers the space performance of massive MIMO based on the heterogeneous network model,designs a first order precoding matrix to eliminate the interference of macro cellular base station to the user of hotspot area.Using the random matrix theory, the spectrum efficiency and energy efficiency of the hot spot area are analyzed. Finally, the relationship between the energy efficiency and the number of base station antennas, the transmit power of the base station is obtained by simulation.

\section{System Model}

We consider a heterogeneous network downlink model: A macrocellbase stationis equipped with $M$ antennas $(M \gg 1)$.in the macrocell cellularcoverage area,there are single antenna users $K$ served.in which $S$ users are concentrated and formed $N$ hotspots.Each hotspot has a micro cellular base station to provide service, equipped with $U$ antennas ( $U<M, U \gg 1$ ). Other $Q$ users served by macrocell and are distributed uniformly in the macro cellular coverage area(not including the hot spot area).

$Q$ single users' received signal vector:

$$
\boldsymbol{y}=\sqrt{P} \boldsymbol{H F x}+\boldsymbol{n}(1)
$$

where $y=\left[y_{1}, y_{2}, \ldots y_{Q}\right]^{T}$ is Q users' received signal vector, $P$ is the transmit power of macrocell and is normalized,i.e., $\mathrm{E}\left\{\|\boldsymbol{x}\|^{2}\right\}=1, \boldsymbol{x}=\left[\boldsymbol{x}_{1}, \boldsymbol{x}_{2}, \ldots, \boldsymbol{x}_{\mathbf{Q}}\right]^{\mathrm{T}}$ is the signal vector thatmacrocell sent to $Q$ 
users. $\boldsymbol{H}=\left[\boldsymbol{h}_{1}, \boldsymbol{h}_{2}, \ldots, \boldsymbol{h}_{\mathbf{Q}}\right]$ is thedownlink channel matrix from macrocellto single users,of dimension $Q \times M . F=\left[\boldsymbol{f}_{1}, \boldsymbol{f}_{2}, \ldots, \boldsymbol{f}_{Q}\right]$ isprecoding matrix, $\boldsymbol{n}$ is the additiveGauss white noise vector of zero mean, $\boldsymbol{n}=\left[\boldsymbol{n}_{1}, \boldsymbol{n} \mathbf{2}, \ldots, \boldsymbol{n}_{\mathbf{Q}}\right]^{\mathrm{T}}$.

If $\boldsymbol{G}=\left[\boldsymbol{g}_{1}, \boldsymbol{g}_{2}, \ldots, \boldsymbol{g}_{Q}\right]^{\mathrm{T}}$ is a complex small-scale fading matrix, $\boldsymbol{D}=\operatorname{diag}\left\{\boldsymbol{d}_{1}, \boldsymbol{d}_{2}, \ldots, \boldsymbol{d}_{\mathbf{Q}}\right\}$ is large scale fading coefficient matrix, $\boldsymbol{H}=\boldsymbol{G} \boldsymbol{D}^{1 / 2}$.It is assumed that single usersnot is affected by the interference from the micro cellular base station, so the signal received by theuser $q$ is gived:

$$
\boldsymbol{y}_{\boldsymbol{q}}=\sqrt{P} \boldsymbol{h}_{\boldsymbol{q}} \boldsymbol{f}_{\boldsymbol{q}} \boldsymbol{x}_{\boldsymbol{q}}+\sqrt{P} \sum_{\substack{i=1 \\ i \neq q}}^{Q} \boldsymbol{h}_{\boldsymbol{q}} \boldsymbol{f}_{\boldsymbol{i}} \boldsymbol{x}_{\boldsymbol{i}}+\boldsymbol{n}_{\boldsymbol{q}}
$$

We assume that users of hotspots are served preferentiallyby micro cellular base stations, the macro cellular base station to the users' signal first to reach the area of micro cellular base station, and then transmit to the users.the user of this hotspot is interfered by the around hotspots, other users of this hotspot.We assume that each hotspot has $S_{n}$ users, thenthe user $s$ of a hotspot received signal is gived

$$
\boldsymbol{y}_{s}=\sqrt{P_{s}} \boldsymbol{b}_{s}^{L} \boldsymbol{f}_{s}^{\boldsymbol{L}} \boldsymbol{x}_{\boldsymbol{s}}+\sqrt{P} \boldsymbol{h}_{s} \boldsymbol{f}_{s} \boldsymbol{x}_{\boldsymbol{s}}+\sqrt{P_{s}} \sum_{\substack{n=1 \\ n \neq L}}^{N} \sum_{s^{\prime}=1}^{S_{n}} \boldsymbol{b}_{s^{\prime} s} \boldsymbol{f}_{s^{\prime}} \boldsymbol{x}_{\boldsymbol{s}^{\prime}}+\sqrt{P_{s}} \sum_{\substack{s^{\prime \prime}=1 \\ s^{\prime \prime} \neq s}}^{s_{n}} \boldsymbol{b}_{s^{\prime \prime} s}^{\boldsymbol{L}} \boldsymbol{f}_{s^{\prime \prime}}^{\boldsymbol{L}} \boldsymbol{x}_{\boldsymbol{s}^{\prime \prime}}+\boldsymbol{n}_{\boldsymbol{s}} \text { (3) }
$$

Where $P_{s}$ is the transmit power of micro cellular base station, $\boldsymbol{b}_{s}^{L}$ and $\boldsymbol{f}_{s}{ }^{L}$ are the channelvector and precoding vector from micro cellular base station to the user $s$,respectively.the second of the formula on the right is the interference vector from macrocell base station. The third is the interference of other regional hotspots to $L$. The fourth is the interference from other users in the region $L$, and $\boldsymbol{n}_{\boldsymbol{s}}$ is the additive Gauss white noise.

\section{Spectral efficiency and energy efficiency analysis}

We mainly consider the spectrum efficiency and energy efficiency of the hotspots, for the users of the hotspots, because of the complexity of the environmentand the interference, we useJointSpatial Division and Multiplexing(JSDM) to eliminate the interference caused by macrocell base station. In order to effectively utilize JSDM and consider the influence of terrain factors, we assume that users in the same hotspots meet one-ring scattering model environment[3], and theeach user's channel covariance matrixhas the same distribution.the channel covariance matrix is mainly determined by the arrival angle and the angular spread, then the hotspots of the channel covariance matrix $(\mathrm{m}, \mathrm{n})$ is expressed as:

$$
[R]_{(m, n)}=\frac{1}{2 \Delta} \int_{\theta-\Delta}^{\theta+\Delta} e^{-j 2 \pi D(m-n) \sin (\alpha)} d_{\alpha}
$$

Where $\mathrm{D}$ is the normalized interval of antenna arrays which relate to the carrier wave length. $R_{L}^{w}$ indicates the covariance matrix from the macrocell base station to the regionL, makingKarhunen-Loeve representation, $\boldsymbol{R}_{L}^{w}=\boldsymbol{U}_{L}^{w} \boldsymbol{\Lambda}_{L}^{w}\left(\boldsymbol{U}_{L}^{w}\right)^{\boldsymbol{H}}$, rank is $\gamma$. Then the interference channel coefficients from the macrocell base station to the user $\mathrm{s}$ of region $\mathrm{L}$ is:

$$
\boldsymbol{h}_{s}=\boldsymbol{A}_{s}^{w}\left(\boldsymbol{\Lambda}_{L}^{w}\right)^{1 / 2} \boldsymbol{U}_{L}^{w}
$$

Where $\boldsymbol{\Lambda}_{L}^{w}$ is the thenonzero covariance eigenvaluesdiagonal matrix. $\boldsymbol{U}_{L}^{w}$ isthe tallunitary matrix of eigenvectors, $\boldsymbol{A}_{s}^{w} \sim \boldsymbol{C N}\left(\mathbf{0}, I_{\gamma}\right)$.

We design a order precoding matrix to eliminate the interference from the macrocellbase station.when micro cellularbase station to estimate the high unitary matrix, $\boldsymbol{U}_{L}^{w}\left(\boldsymbol{U}_{L}^{w}\right)^{\boldsymbol{H}}=\boldsymbol{I}_{\gamma}$, we design precoding matrix vector $\boldsymbol{f}_{s}=\left(\boldsymbol{U}_{L}^{w}\right)^{\boldsymbol{H}}$, so macrocell base station interference are eliminated.Therefore,the spectrum efficiency of the hotspot $L$ is: 


$$
\sum_{s=1}^{S} R=\sum_{S=1}^{S} E\left\{\log _{2}\left(1+\frac{\left.\left.\frac{P_{S}}{S}\right|_{\boldsymbol{s}} ^{\boldsymbol{L}} \boldsymbol{f}_{\boldsymbol{s}}^{\boldsymbol{L}}\right|^{2}+\frac{P}{K}\left|\boldsymbol{h}_{\boldsymbol{s}} \boldsymbol{f}_{\boldsymbol{s}}\right|^{2}}{\frac{P_{S}}{S} \sum_{i=1, i \neq s}^{S_{n}}\left|\boldsymbol{b}_{\boldsymbol{s}^{\prime \prime} \boldsymbol{s}}^{\boldsymbol{L}} \boldsymbol{f}_{\boldsymbol{s}^{\prime \prime}}^{\boldsymbol{L}}\right|^{2}+\frac{P_{S}}{S} \sum_{n=1, n \neq L}^{N} \sum_{\boldsymbol{s}^{\prime}=1}^{S_{n}}\left|\boldsymbol{b}_{\boldsymbol{s}^{\prime} \boldsymbol{s}} \boldsymbol{f}_{\boldsymbol{s}^{\prime}}\right|^{2}+1}\right)\right\}
$$

each micro cellular region bases on zero forcing(ZF) precoding algorithm to eliminate the interference in the region, ZF precoding matrix of each micro cellular base station is $\boldsymbol{F}_{s}=\boldsymbol{B}_{s}^{H}\left(\boldsymbol{B}_{s} \boldsymbol{B}_{s}^{H}\right)^{-1}$, where $\boldsymbol{B}_{s}$ is the channel matrix of each micro cellular base station to the user. Transmit power of the micro cellular base station is $P_{s}=E_{s} / U$ ( $E_{s}$ is the single antenna transmit power of themicro cellular base station, the macrocell transmit power.Make $\boldsymbol{F}_{s}=\frac{\boldsymbol{B}_{s}^{\boldsymbol{H}}}{U}\left(\frac{\boldsymbol{B}_{s} \boldsymbol{B}_{s}^{\boldsymbol{H}}}{U}\right)^{-1}$, unitization $\overline{\boldsymbol{F}_{s}}=\left[\boldsymbol{f}_{1}, \boldsymbol{f}_{2}, \ldots \boldsymbol{f}_{s}\right]$,

where $\boldsymbol{f}_{s}=\frac{\boldsymbol{b}_{s}^{\boldsymbol{H}}}{\left\|\boldsymbol{b}_{s}\right\|}$. Then (6) can be written as

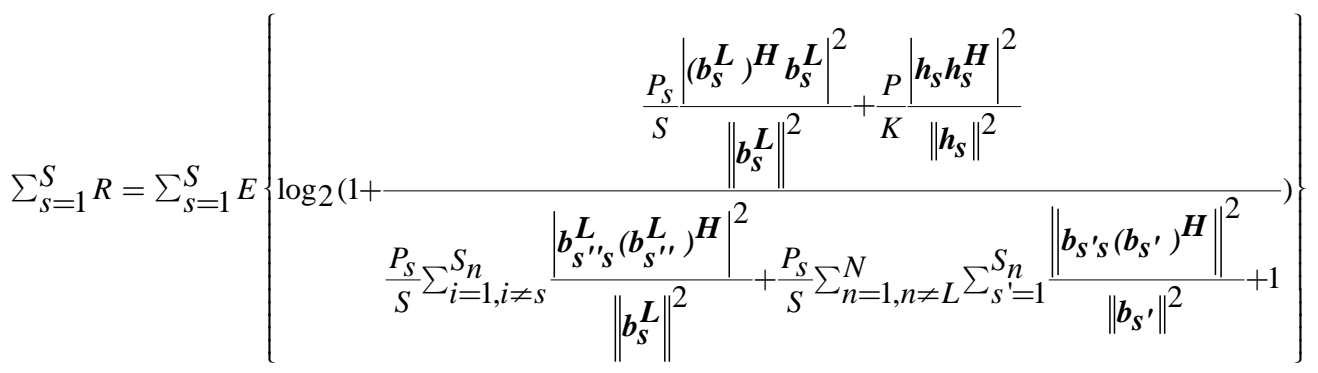

According to the random matrix theory of the literature [4], when $U \rightarrow \infty, M \rightarrow \infty$,

$$
\begin{aligned}
& \frac{\left.\mid \boldsymbol{b}_{s}^{L}\right)\left.^{H} \boldsymbol{b}_{s}^{L}\right|^{2}}{U\left\|\boldsymbol{b}_{s}^{L}\right\|^{2}}=\beta^{2}, \frac{\left|\boldsymbol{h}_{s} \boldsymbol{h}_{s}^{H}\right|^{2}}{M\left\|\boldsymbol{h}_{s}\right\|^{2}}=d_{s}, \frac{\left|\boldsymbol{b}_{s^{\prime \prime} s}^{L}\left(\boldsymbol{b}_{s^{\prime \prime}}^{L}\right)^{H}\right|^{2}}{U\left\|\boldsymbol{b}_{s}^{L}\right\|^{2}}=0, \frac{\left|\boldsymbol{b}_{s^{\prime} s} \boldsymbol{b}_{s^{\prime}}^{\boldsymbol{H}}\right|}{U\left\|\boldsymbol{b}_{s^{\prime}}\right\|^{2}}=0 \\
& \text { Then, } \sum_{s=1}^{S} R=\sum_{s=1}^{S} E\left\{\log _{2}\left(1+\frac{E_{s}}{S} \beta_{s}+\frac{E}{K} d_{s}\right)\right\}=\sum_{s=1}^{S} E\left\{\log _{2}\left(1+\frac{P_{s} U}{S} \beta_{s}+\frac{P M}{K} d_{s}\right)\right\}, \\
& U \rightarrow \infty, M \rightarrow \infty .
\end{aligned}
$$

\section{4. simulation}

Simulation environment: single antenna users $K=20, S=10$, there are two hot spots, micro cellular base station antennas $U=0.5 \mathrm{M}$, micro cellular base stationtransmit power $P_{s}=0.5 P$, $\beta_{s}=0.3 . d_{s}=0.5$,Fig. 1 report when macrocell base station transmitting power $P$ fixed,M=50,100,200,300,400,500, respectively,the change of $\eta$.Fig.2 presents when the macro cellular transmit antenna fixed and macrocell base station power is 5,10,15,20,30,respectively,the change of $\eta$.

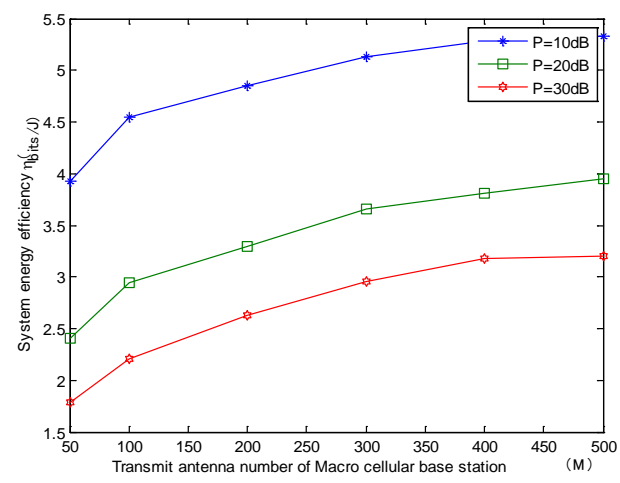

Fig.1

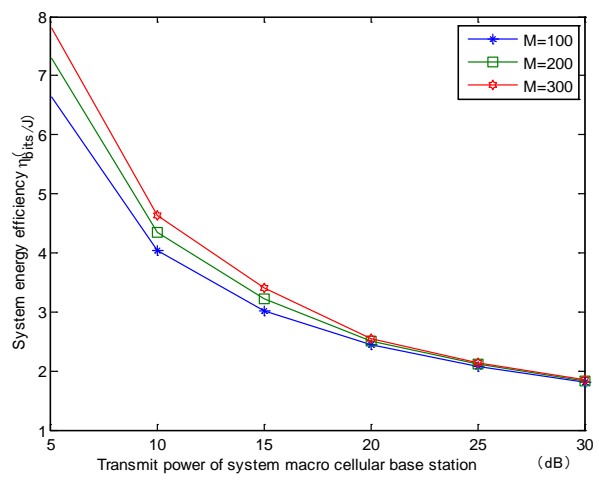

Fig.2 
From the Fig.1,the energy efficiency of the hotspot increaseswith increasing of the number of the antennas of macrocellbase station, but the magnitude of the increaseisdecreasing ,finally, it tends to be stable. At the same time, it can be shown on the Fig.2 that the energy efficiency of the hot spot decreases with the increase of the transmission power of the macro cell base station,and the effect of the number of the macro cellular base station antennas on the energy efficiency is very low when the transmit power reaches a certain value.

\section{Summary}

In this paper, we provide a design on the energy efficiency of a heterogeneous network with Massive-MIMO.the network is optimized by using JointSpatial Division and Multiplexing(JSDM) and zero forcing algorithm.Simulationresults show that heterogeneous networks with Massive-MIMO can improve the energy efficiency of the system,

\section{References}

[1].E. G. Larsson, O. Edfors, F. Tufvesson, and T. L. Marzetta. MassiveMIMO fornext generation wireless systems.IEEE CommunicationsMagazine, Vol. 52(2014),No. 2, p. 186-195.

[2].F. Rusek, D. Persson, B. K. Lau, E. G. Larsson, T. L.Marzetta,O. Edfors, and F. Tufvesson. Scaling up MIMO : Opportunities andchallenges with very large arrays. IEEE Signal Processing Magazine.Vol. 30(2013), No.1, p. 40-60.

[3].AnsumanAdhikary, Harpreet S. Dhillon,GiuseppeCaire.Massive-MIMO Meets HetNet: InterferenceCoordination Through Spatial Blanking.IEEE Journals \& Magazines. Vol. 33(2015), No.6, p. 1171-1186.

[4]. HienQuoc Ngo, Erik G. Larsson, Thomas L. Marzetta.Energy and Spectral Efficiency ofVery Large Multiuser MIMO Systems.IEEETransaction on Communications. Vol. 61(2013), No.4, p. 1436-1449. 